\title{
Effect of laser scribing on grain-oriented silicon steel
} Wenzhou Tian ${ }^{1,2, a}$, Yechao Zhu ${ }^{2,3, b}$, Zexi Yuan ${ }^{1, c}, Z o n g h a n L^{2,3, d}$

${ }^{1}$ Wuhan University of Science and Technology,Wuhan,430080,China

${ }^{2}$ Wuhan Iron and Steel (Group) Corp.Wuhan,430080,China

${ }^{3}$ National Silicon Steel Engineering Research Center,Wuhan,430081,China

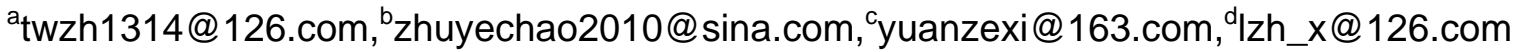

Keywords: Grain-oriented silicon steel, Laser scribing, Coating

\begin{abstract}
Laser scribing is widely used to reduce core loss of grain-oriented silicon steel recently. The effects of laser scribing on surface coating and magnetic properties of silicon steel were investigated in this paper. The effect of stress annealing treatment on laser scribing product has been analyzed. It was found that as laser power increased, the core loss reduced and the damage to surface coating exacerbated. Reducing the laser scribing line spacing to a certain width can achieve low core loss. The core loss of laser domain refined product will increase after annealing at certain temperature.
\end{abstract}

\section{Introduction}

Grain-oriented silicon steel is widely used for transformer cores, and reducing of iron loss is one of the most important industrial issues. The grain-oriented silicon steel core loss consists mainly of hysteresis loss and eddy current losses.

As the improvement of grain-oriented silicon steel sheet manufacturing technology, abnormal eddy current losses become the mainly composition which is about $70 \%$ of total core loss on high magnetic grain-oriented silicon steel. ${ }^{[1]}$ Abnormal eddy current loss is based on the eddy current loss of the magnetic domain wall movement, and is proportional to the rate of movement of $180^{\circ}$ magnetic domain wall. Under the same frequency, the domain wall moving speed is proportional to the distance moved, so the larger magnetic domain width, the more core loss. As is known, it's very effective for refining magnetic domain wall spacing and hence reducing iron loss. ${ }^{[2]}$

In particular, the magnetic domain-refining techniques, such as laser scribing, groove-forming and etching techniques have been developed since nearly 30 years. ${ }^{[3]}$ However, the laser scribing is the main technique used in industry which the direction of scribing usually $90^{\circ} \mathrm{C}$ from rolling direction. It has been speculated that the induced tensile stresses or recrystallized micro grains might be the origin of the magnetic domain refining. ${ }^{[3,4]}$ Though laser scribing can reduce core loss effectively, silicon steel coating surface is usually damaged by the pulse laser and the effect of core loss reducing can't reserve after annealing treatment. ${ }^{[1-7]}$

\section{Experimental}

The continuous high-speed scanning system with $\mathrm{CO}_{2}$ laser was used to scribe line on grain-oriented silicon steel surface along the direction vertical to rolling. The morphology of scribing line was observed by Scanning Electron Microscope (SEM ) and the contents inside and outside of scribing line were analyzed using Energy Dispersive Spectrometer (EDS). In this study, high magnetic induction grain-oriented silicon steel sheets of $500 \times 500 \times 0.27 \mathrm{~mm}^{3}$ were used as the specimens to measure magnetic properties. Laser scribing samples were annealed at $400^{\circ} \mathrm{C}, 500^{\circ} \mathrm{C}, 600^{\circ} \mathrm{C}, 700^{\circ} \mathrm{C}$ and $800^{\circ} \mathrm{C}$ using box-type electrical resistance furnace with the protective atmosphere $70 \% \mathrm{~N}_{2}+30 \% \mathrm{H}_{2}$ for 2 hours. The SST-500 type Silicon Steel AC Magnetic Measuring Instrument made by Chinese National Institute of Metrology was used to measure magnetic properties in this paper. Rate of magnetic properties improvement: $\Delta \mathrm{B}_{800} / \mathrm{P}_{17 / 50}=\left(\mathrm{B}_{800} / \mathrm{P}_{17 / 50}\right.$ (before laser scribing) - $\mathrm{B}_{800} / \mathrm{P}_{17 / 50}$ (after laser scribing)) / ( $\mathrm{B}_{800} / \mathrm{P}_{17 / 50}$ (before laser scribing) $) \times 100 \%$. 


\section{Results and discussion}

\section{Laser scribing effect on coating surface.}

Fig. 1 shows the morphology of the laser scribing line of high magnetic grain-oriented silicon steel which scribing line spacing is $\mathrm{L}_{\mathrm{n}}$ (thickness $=0.27 \mathrm{~mm}, \mathrm{~B}_{800}=1.931(\mathrm{~T}), \mathrm{P}_{17 / 50}=0.956(\mathrm{w} / \mathrm{kg})$; before laser scribing ).Fig. 2 shows the Fe content of coating surface near the scribing line in different laser power.

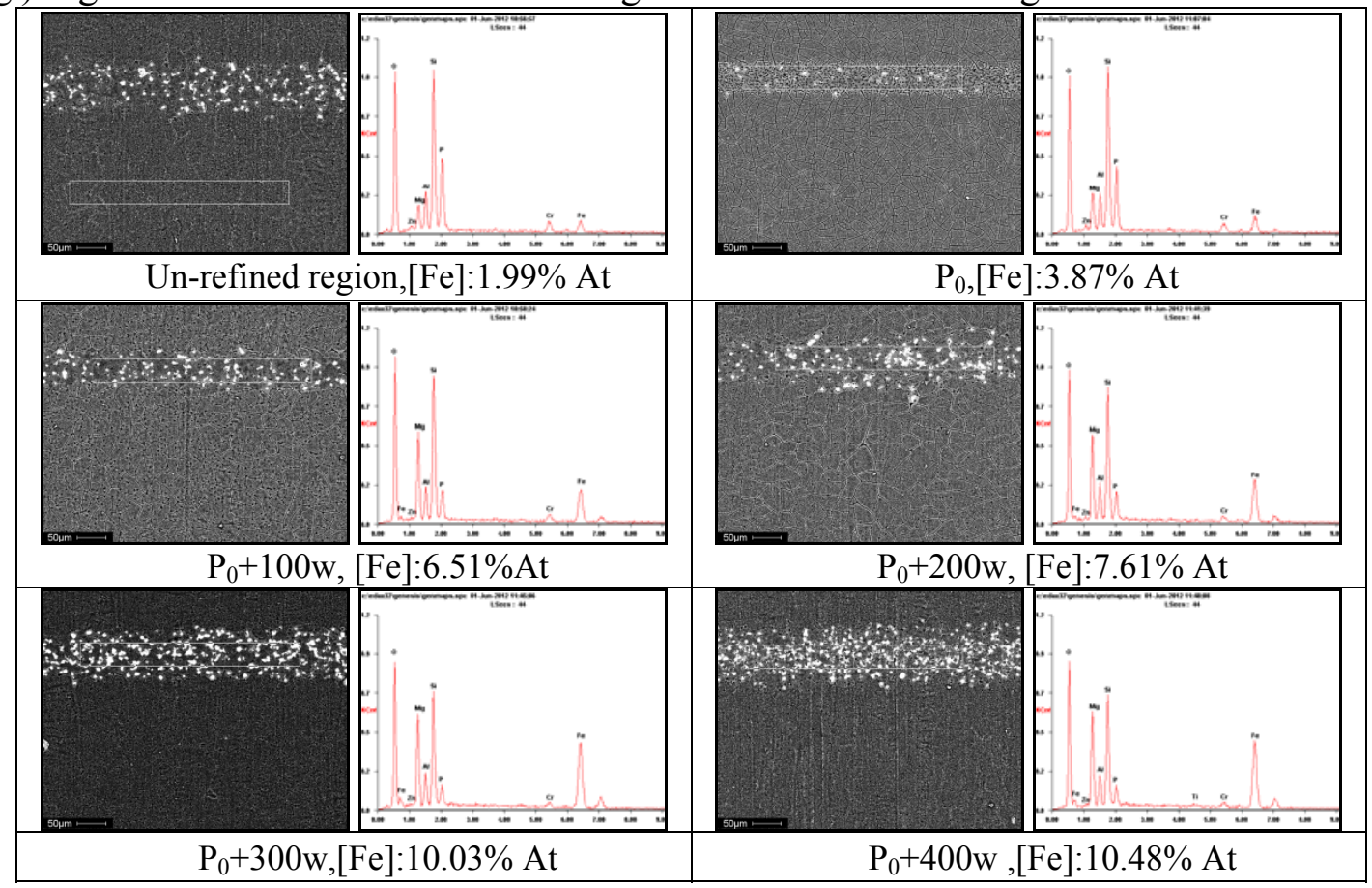

Fig.1. Morphology and composition near the refine line in different laser power

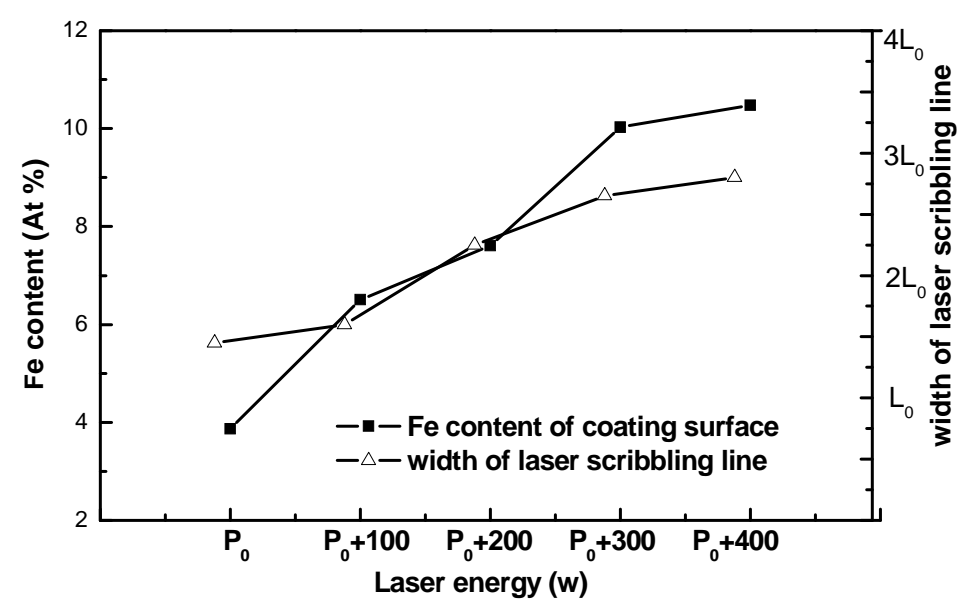

Fig.2. Fe content and width of scribing line in different laser power

Grain-oriented silicon steel has formed a layer of insulating coating on the surface before laser scribing. It can estimate the degree of damaging coating by analyzing the width of scribing line and Fe content within the line.Fig.1 and Fig.2 shows all specimens' coating has damaged by laser beam in different degree. The lasers power more higher, the more width of scribing line and the more much $\mathrm{Fe}$ content within scribing line. Laser power $\mathrm{P}_{0}$, the scribing line width is about $30 \mu \mathrm{m}$ and $[\mathrm{Fe}]$ is $3.87 \%$; when laser power increased $300 \mathrm{w}$, the line width become about $50 \mu \mathrm{m}$ and [Fe] increased to $10.03 \%$. The laser power to further increase, the width of line has no significant change. If the laser power is increased more $200 \mathrm{w}$, the damaging of coating become serious and Fe content of coating surface increased rapidly. As is to know, energy and holding time within line zone of laser beam are main factor which damaged coating, and the former decided by laser power, the later decided by laser scanning speed. ${ }^{[5,6]}$ At the same laser scanning speed, laser power is the most significant factor. 


\section{Laser scribing effect on magnetic property.}

The desired domain refining results can achieved by adjusting scribing line spacing when choose suitable laser power, while not damage coating serious. If laser power is too big, the reduction of eddy current loss will be offset by increased hysteresis loss addition to damaging coating. ${ }^{[6]}$

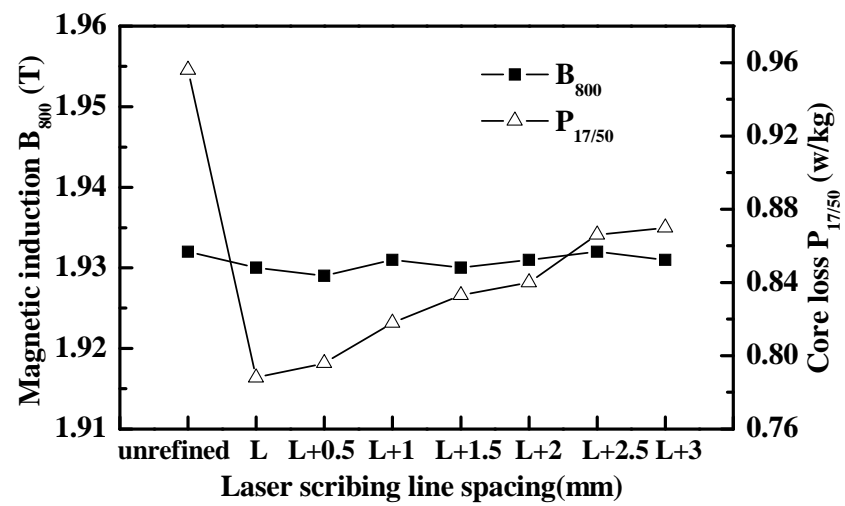

Fig.3.The effect of laser scribing line spacing on magnetic properties

Fig. 3 shows the effect of laser scribing line spacing on the rate of core loss and magnetic induction improvement (laser power: $\mathrm{P}_{0}+100 \mathrm{w}$ ). With the narrowing of scribing line spacing, magnetic induction changed little and core loss reduced significantly. While the scribing line spacing narrow than $\mathrm{L}+2.5 \mathrm{~mm}$, the rate of core loss improvement increase substantially. However, if the spacing narrow than $\mathrm{L}+1 \mathrm{~mm}$, the changing of core loss became little. The results mainly because of laser scribing line spacing value close to the domain width threshold. ${ }^{[5]}$

\section{Annealing treatment effect on laser domain refined product.}

Fig.4. shows the variation of magnetic induction and core loss affect with annealing temperature. Annealed at different temperatures, the sample magnetic induction changes of small but iron loss vary within wide limits $\left(0.27 \mathrm{~mm}, \mathrm{~B}_{800}: 1.931 \mathrm{~T}, \mathrm{P}_{17 / 50}: 0.956 \mathrm{w} / \mathrm{kg}\right.$; before laser scribing $)$.

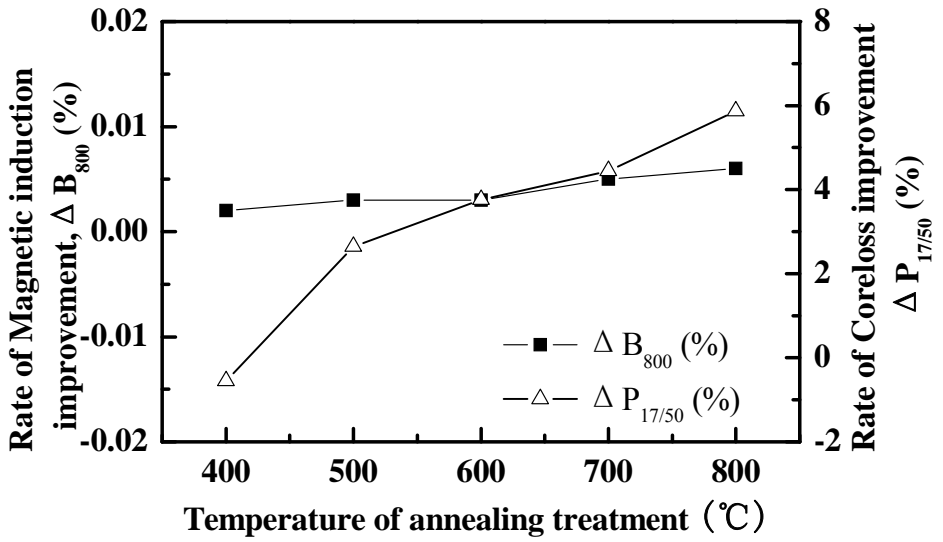

Fig.4.The variation of magnetic properties affect with annealing temperature.

Sample core loss reduced slightly annealing at $400{ }^{\circ} \mathrm{C}$, but it increased significantly when annealing temperature is more than $500^{\circ} \mathrm{C}$. The annealing can lighten the adverse effects of residual stress and shear stress on magnetic properties. At the meantime, annealing treatment can weaken or eliminate domain refinement effects arising from the tensile stress. And so, core loss reduction caused by laser scribing is weaken and even eliminated.Fig.4 indicate annealing treatment more than $400^{\circ} \mathrm{C}$ have almost the same effect on magnetic induction, it may because of the annealing at $400^{\circ} \mathrm{C}$ to $800^{\circ} \mathrm{C}$ could not change the Gauss-grains deviation angle. 


\section{Conclusions}

Laser scribing is the usually means of reducing core loss in the large-scale industrial producing grain-oriented silicon steel, when laser energy increased, the core loss reduced more and the damage of coating surface exacerbated.

Controlling the damage to coating surface by chooses a suitable laser power, and it can achieve optimal iron loss reduction by decreasing the laser scribing line spacing to a certain width.

The core loss of laser scribing domain refined product will increase after annealing treatment on certain temperature, and it sharp increased when the annealing temperature more than $500^{\circ} \mathrm{C}$ 。

\section{References}

[1]He zongzi: Electric Steel (Metallurgical Industry Publications, Beijing 1996)

[2]M.Enokizono, I.Tanabe,etc: Effects of scratching on two-dimensional magnetic properties in grain-oriented silicon steel sheet. Journal of Magnetism and Magnetic Materials 196-197 (1999) 335-337

[3]Muneyuki Imafuku, Hiroshi Suzuki, etc: Effects of laser irradiation on iron loss reduction for Fe-3\%Si grain-oriented silicon steel. Acta Materialia 53(2005) 939-945

[4]Jingdong Li, Yuqin Gu, Zenyuan Guo: Decreasing the core loss of grain-oriented silicon steel by laser processing. Journal of Materials Processing Technology 69(1997)180-185

[5]ZHU Yechao, WANG Liangfang, QIAO Xueliang: Methods and mechanism of the domain refinement of grain oriented silicon steel by surface treatment. Research on Iron \& Steel, Dec 2006, Vol.34, No.6

[6]G.C.Rauch, R.F.Krause: Effect of beam dwell time on surface changes during laser scribing. J.Appl.Phys. 57(1), 15April 1985

[7]Jingdong Li, Yuqin Gu, Zenyuan Guo: Analysis of the phenomena of non-Fourier heat conduction in switch-Q laser processing for reducing the core loss of grain-oriented silicon steel. Journal of Materials Processing Technology 74(1998)292-297 